\title{
Mudanças florísticas e estruturais na comunidade arbórea de uma floresta de vale no Parque Nacional da Chapada dos Guimarães, Mato Grosso, Brasil ${ }^{1}$
}

\author{
JOSÉ ROBERTO R. PINTO ${ }^{2,4}$ e JOHN DU VALL HAY 3
}

(recebido: 12 de março de 2004; aceito: 2 de junho de 2005)

\begin{abstract}
Changes in the floristics and structure in the tree community in a valley forest of the Chapada dos Guimarães National Park, Mato Grosso State, Brazil). The objective of this study was to describe the changes in the floristic composition and community structure of the tree-shrub layer in the valley forest in the Chapada dos Guimarães National Park in Mato Grosso State, Brazil. The data were collected in three parallel transects, systematically distributed and equidistant on both sides of the stream. The tree layer (diameter at the breast height $\geq 5 \mathrm{~cm}$ ) was surveyed in 1996 and 1999 in 18 plots of $600 \mathrm{~m}^{2}$ each. The saplings (diameter at the breast height $\geq 1 \mathrm{~cm}$ and $<5 \mathrm{~cm}$ ) and juveniles (total height $\geq 30 \mathrm{~cm}$ and diameter at the base of at least $<1 \mathrm{~cm}$ ) were surveyed in 1999 and 2001 in 36 plots of 50 and $6 \mathrm{~m}^{2}$ respectively. The high species richness, a total of 212 species, was probably due to the inclusion of saplings and juveniles along with the tree layer. The change in floristic composition was small and gains and losses were limited to those species with low abundance ( $<3$ individuals). These changes did not reflect significant changes in diversity. The size distribution, height and diameter, did not differ between sampling dates indicating a relative stability in this forest. However the apparent floristic and community stability should not be interpreted as indicating that the forest is at climax since changes are occurring in both time and space.
\end{abstract}

Key words - community dynamics, demographic changes, diversity changes, floristic changes

RESUMO - (Mudanças florísticas e estruturais na comunidade arbórea de uma floresta de vale no Parque Nacional da Chapada dos Guimarães, Mato Grosso, Brasil). O objetivo deste estudo foi descrever as alterações na composição florística e na estrutura da comunidade arbóreo-arbustiva da floresta de vale do Véu de Noiva, Parque Nacional da Chapada dos Guimarães, Mato Grosso. O estudo foi conduzido em três transeções paralelas, distribuídas de forma sistemática e eqüidistante, em ambas vertentes do vale. Para árvores (diâmetro à altura do peito - DAP $\geq 5 \mathrm{~cm}$ ) o levantamento foi realizado em 1996 e $1999 \mathrm{em} 18$ parcelas de $600 \mathrm{~m}^{2}$, e para as arvoretas $(1 \geq \mathrm{DAP}<5 \mathrm{~cm}$ ) e juvenis (altura $\geq 30 \mathrm{~cm}$ e DAP $<1 \mathrm{~cm}$ ) em $1999 \mathrm{e} 2001 \mathrm{em} 36$ subparcelas de 50 e $6 \mathrm{~m}^{2}$, respectivamente. A alta riqueza florística registrada (212 espécies) está associada ao fato de terem sido computados juntos as árvores, arvoretas e juvenis. A mudança na composição florística foi pequena, com perdas e ganhos se limitando às espécies amostradas com baixa abundância ( $<3$ indivíduos). Estas alterações não refletiram em mudanças significativas nos índices de diversidade. As distribuições de indivíduos nas classes de diâmetro e altura não diferiram significativamente entre os anos, indicando que a estrutura se manteve estável. No entanto, a aparente estabilidade florística e estrutural não deve ser interpretada como sendo a floresta do Véu de Noiva uma comunidade estática, pois mudanças estão acontecendo constantemente ao longo do tempo e espaço.

Palavras-chave - dinâmica de comunidade, mudanças demográficas, mudanças florísticas, mudanças na diversidade

\section{Introdução}

Os modernos estudos realizados nos ambientes tropicais nas últimas décadas têm proporcionado grandes avanços no entendimento dos processos ecológicos que regem a natureza, principalmente nos ecossistemas

1. Parte da tese de doutorado do primeiro autor em Ecologia, UnB.

2. Universidade de Brasília, Departamento de Engenharia Florestal, Caixa Postal 04357, 70919-970 Brasília, DF, Brasil.

3. Universidade de Brasília, Departamento de Ecologia, Campus Darcy Ribeiro, 70919-970 Brasília, DF, Brasil.

4. Autor para correspondência: jrrpinto@unb.br florestais (Hartshorn 1990). No entanto, a maioria dos autores apresenta e discute os resultados com base em dados obtidos a partir de um único momento. Na maioria das vezes, estes estudos geralmente não consideram os aspectos relacionados às mudanças florísticas e estruturais das comunidades ao longo do tempo (Werneck et al. 2000).

Estudos envolvendo duas ou mais observações de campo numa mesma área geralmente apontam a dinâmica de clareiras e a heterogeneidade ambiental como os principais fatores responsáveis pela manutenção da alta diversidade de espécies nas florestais tropicais (Rees et al. 2001). Por um lado, os estudos de clareiras normalmente se limitam a 
investigar as diferenças bióticas e abióticas entre as áreas de clareira e não clareira (Lieberman et al. 1989) e falham na tentativa de elucidar o paradoxo da diversidade de espécies em ecossistemas florestais. Por outro lado, a heterogeneidade ambiental têm sido invocada na grande maioria dos trabalhos como o mais relevante fator responsável pela ocorrência da alta diversidade biológica nos ecossistemas tropicais (Shugart 1984, Swaine 1990, Whitmore 1990, Richards 1996).

Segundo Tilman et al. (1997), a interação entre o meio biótico e a dinâmica da vegetação determina as principais características das comunidades de plantas, inclusive sua composição florística. No entanto, estudos de dinâmica de comunidades são essenciais para o entendimento dos padrões de riqueza de espécies (Phillips et al. 1994), haja visto que mudanças nas taxas demográficas da comunidade podem afetar a composição florística e a estrutura futura das florestas (Phillips \& Gentry 1994, Felfili 1995a). Dentro deste contexto, os ecossistemas ripários oferecem boas oportunidades de se avaliar como a diversidade de espécies e a estrutura das comunidades variam nas escalas espacial e temporal (Naiman \& Décamps 1997), pois estes apresentam grande heterogeneidade ambiental, tanto no sentido longitudinal como perpendicular ao curso d'água (Ribeiro \& Walter 2001, Rodrigues \& Nave 2000).

Estudos de dinâmica da vegetação no Brasil são recentes e escassos, exceto para a região Amazônica (Felfili 2000, Oliveira Filho 2002) e poucas informações encontram-se disponíveis sobre as mudanças florísticas e estruturais das comunidades arbóreo-arbustivas. Esta escassez de informações evidencia a necessidade urgente de se obter dados sobre a variação temporal em florestais nativas. Assim, além de incrementar o conhecimento sobre a dinâmica das florestas tropicais, o objetivo deste estudo foi analisar as alterações na composição florística, na diversidade e na estrutura da comunidade arbóreo-arbustiva em uma floresta de vale, localizada no Parque Nacional da Chapada dos Guimarães, Mato Grosso, Brasil.

\section{Material e métodos}

Área de estudo - A área estudada está localizada no Parque Nacional da Chapada dos Guimarães (PNCG), Mato Grosso, Brasil (figura 1). O Parque possui uma área de 32.670 ha e consiste em uma importante zona de transição entre o Cerrado e a Floresta Amazônica, situado entre as latitudes $15^{\circ} 12^{\prime} \mathrm{S}$ e $15^{\circ} 30^{\prime} \mathrm{S}$ e as longitudes $56^{\circ} 45^{\prime} \mathrm{W}$ e $56^{\circ} 50^{\prime} \mathrm{W}$, com altitudes

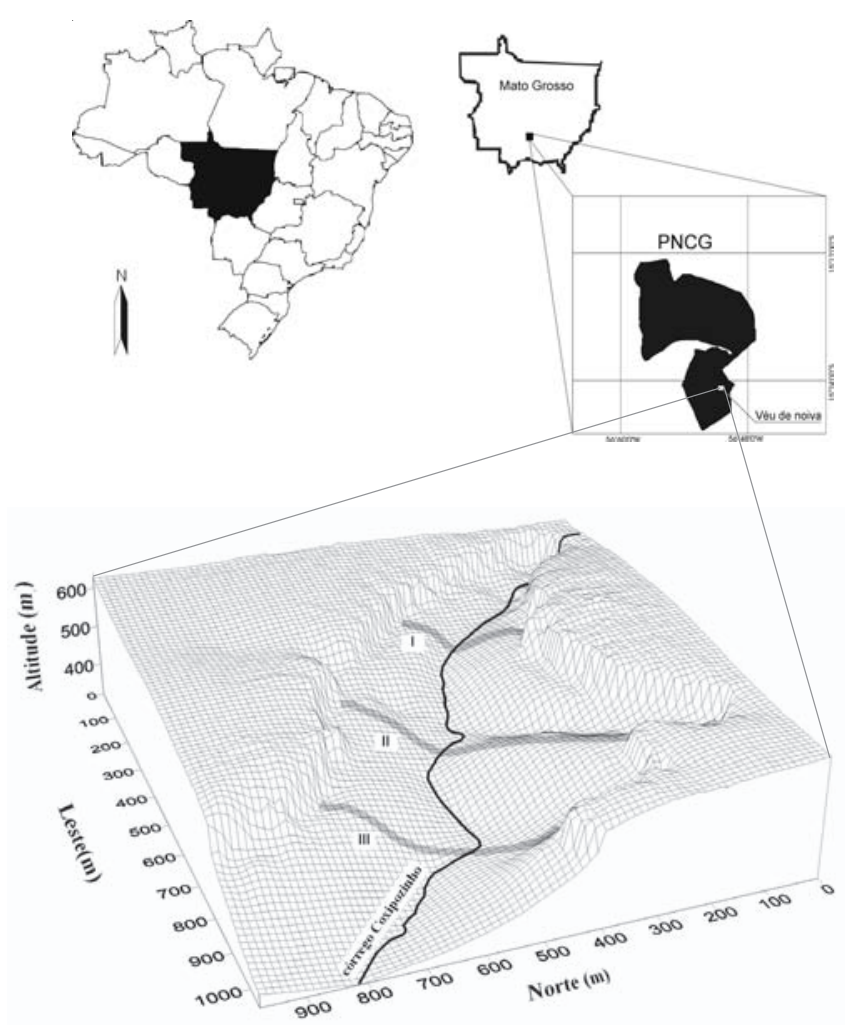

Figura 1. Localização geográfica do Parque Nacional da Chapada dos Guimarães (PNCG), Mato Grosso, Brasil e perfil tridimensional da superfície do vale do Véu de Noiva, representando a topografia da área e indicando as três transeções amostradas (I, II e II).

Figure 1. Geographic location of the Chapada dos Guimarães National Park (PNCG), Mato Grosso State, Brazil and three dimensional profile of the surface of the Véu de Noiva valley, showing the topography of the area and the sampled three transects (I, II, and III).

variando de $350 \mathrm{~m}$ na baixada Cuiabana a $800 \mathrm{~m}$ no alto da Chapada (Ibama 1994).

No PNCG predominam os tipos climáticos Aw e Cw, segundo a classificação de Köppen (Ibama 1994), com precipitação média anual variando de 1.800 a $2.000 \mathrm{~mm}$ e temperatura média anual em torno de $22,8^{\circ} \mathrm{C}$, em julho, a $27,2^{\circ} \mathrm{C}$, em outubro (Oliveira Filho \& Martins 1986, Monteiro 1994). Os solos são geralmente classificados como Neossolos quartizarênicos distróficos nos vales e encostas e Latossolos vermelho-amarelo distróficos nas planícies e planaltos (Brasil 1982). A cobertura vegetal é formada pelas diferentes fitofisionomias do Cerrado sensu lato, pelas formações florestais, representadas pelas florestas semidecíduas, decíduas e de galeria; e pela vegetação de altitude, representada pelos campos rupestres (Veloso et al. 1991, Ibama 1994).

A floresta estudada está encaixada no vale situado logo após a queda d'água Véu de Noiva, formada pelo córrego 
Coxipozinho (figura 1). O vale está alojado em uma depressão de aproximadamente $70 \mathrm{~m}$ em relação às áreas adjacentes e ocupa aproximadamente 30 ha (Pinto \& Oliveira Filho 1999). Em geral o solo é raso e com constantes afloramentos rochosos, onde predominam os Neossolos (Litólicos), sendo que manchas de Neossolos quartizarênicos e Neossolos flúvicos também podem ocorrer (Pinto 1997). Como a área de estudo se estende desde o talvegue, parte mais baixa da encosta, até o paredão rochoso, parte mais alta da encosta, é difícil separar fisionomicamente o que é floresta de galeria e o que é floresta de encosta. Neste caso, a denominação 'floresta de vale' (sensu Eiten 1994) é a mais indicada (Pinto \& Oliveira Filho 1999). Portanto, a área estudada neste trabalho foi denominada como "floresta de vale do Véu de Noiva".

Metodologia - O processo de amostragem seguiu o desenho amostral adotado por Pinto \& Oliveira Filho (1999). Em síntese, o estudo foi conduzido em três transeções paralelas distribuídas de forma sistemática e eqüidistante, nas seguintes seções do vale: próximo a queda d'água, no meio do vale e na extremidade oposta à queda d'água (figura 1).

Cada transeção foi formada por seis unidades amostrais instaladas em três setores topográficos, em ambas vertentes do vale: beira do córrego, meio e alto da encosta. As unidades da beira do córrego foram instaladas o mais próximo possível do curso d'água, as do alto da encosta adjacente à escarpa rochosa que delimita o vale e as do meio da encosta posicionada entre as duas extremidades: curso d'água escarpa rochosa. O tamanho das parcelas variou de acordo com as categorias de desenvolvimento das plantas, definidas como: árvores, arvoretas e juvenis. A categoria das árvores correspondeu aos indivíduos com diâmetro à altura do peito (DAP) $\geq 5 \mathrm{~cm}$, medido a $1,30 \mathrm{~m}$ do solo. As arvoretas foram representadas pelos indivíduos com DAP $\geq 1 \mathrm{~cm} \mathrm{e}<5 \mathrm{~cm}$ e as juvenis com altura total $\geq 30 \mathrm{~cm}$ e diâmetro à altura do solo (DAS) $<1 \mathrm{~cm}$. Cabe ressaltar que nesta última categoria foram encontrados alguns indivíduos já em fase reprodutiva; portanto, o termo juvenil, neste caso, se refere ao estágio de desenvolvimento quanto ao porte e não quanto à maturidade reprodutiva.

A categoria das árvores foi amostrada em 18 parcelas de $600 \mathrm{~m}^{2}(20 \times 30 \mathrm{~m})$ nos setores meio e alto da encosta e $10 \times$ 60 m no setor beira do córrego (Pinto e Oliveira Filho 1999). As categorias das arvoretas e juvenis foram registradas em unidades amostrais compostas e formadas por duas subparcelas cada, $5 \times 10$ e $2 \times 3 \mathrm{~m}$, respectivamente (Pinto 2002). As subparcelas foram alocadas de forma sistemática no interior das parcelas de maior tamanho, sucessivamente, conforme adotado por Felfili (1997a).

Para as árvores, o primeiro inventário foi realizado em 1996 e teve como objetivo o levantamento florístico e a análise de gradiente desta floresta de vale (Pinto 1997). O segundo inventário foi realizado três anos depois, em 1999, com a remedição de todas as parcelas. Neste levantamento foram reavaliadas as árvores sobreviventes e amostrados os novos indivíduos que atingiram o diâmetro mínimo de inclusão
(DAP $\geq 5 \mathrm{~cm}$ ). As arvoretas e juvenis foram mensuradas em 1999 e 2001, seguindo o mesmo procedimento adotado para as árvores, porém respeitando os critérios de inclusão.

Todas as espécies registradas pela primeira vez na área foram coletadas, identificadas e o material botânico testemunho depositado nos Herbários da Universidade Federal de Mato Grosso (HCUFMT) e da Universidade de Brasília (UB). A identificação botânica foi feita por meio de comparações em herbário, consulta à literatura especializada e aos especialistas. A classificação botânica foi baseada no sistema APG II (The Angiosperm Phylogeny Group 2003).

Para cada categoria de desenvolvimento e época de levantamento foi calculado o valor de importância (VI), por meio da soma dos valores relativos de densidade, freqüência e dominância (Mueller-Dombois \& Ellenberg 1974); a riqueza, por meio do número de espécies; a diversidade, por meio do índice de Shannon-Wiener ( $\left.\mathrm{H}^{\prime}\right)$; a eqüabilidade, com base no índice de Pielou (J'), de acordo com Magurran (1988). A estrutura vertical e horizontal da comunidade foi descrita em termos de número de indivíduos e área basal, cálculo da mediana em altura e diâmetro, além da distribuição de freqüência nas classes de altura e de diâmetro.

Para comparar o índice de diversidade entre os anos de levantamentos foi utilizado o teste de $t$ para amostras independentes, de acordo com Magurran (1988). A comparação da distribuição de freqüência nas classes de diâmetro e de altura, entre os levantamentos, foi feita por meio do teste de Kolmogorov-Smirnov (Zar 1998), com os indivíduos agrupados em classes de altura e de diâmetro, com amplitude fixa. Para comparar a densidade e a área basal entre os anos inventariados utilizou-se o teste de aderência de Qui-quadrado (Zar 1998).

\section{Resultados e Discussão}

Composição florística e diversidade de espécies Durante o período estudado foram amostrados 3.045 indivíduos pertencentes a 212 espécies, 138 gêneros e 59 famílias, não incluindo o grupo das espécies não identificadas (NI), que foi registrado apenas no primeiro inventário na categoria das arvoretas. Analisando por categoria de desenvolvimento, 1.323 indivíduos foram amostrados como árvores e distribuídos em 150 espécies; 823 como arvoretas em 117 espécies e 899 como juvenis em 110 espécies. A lista das espécies com suas respectivas famílias botânicas, densidade absoluta e valor de importância (VI), nas três categorias e nos dois anos inventariados é apresentada na tabela 1.

O considerável aumento na riqueza florística, de 148 espécies amostradas dentro das parcelas em 1996 (Pinto \& Oliveira Filho 1999) para 212 espécies em 2001, certamente está associado à inclusão das categorias das arvoretas e juvenis na amostragem. A percentagem de 


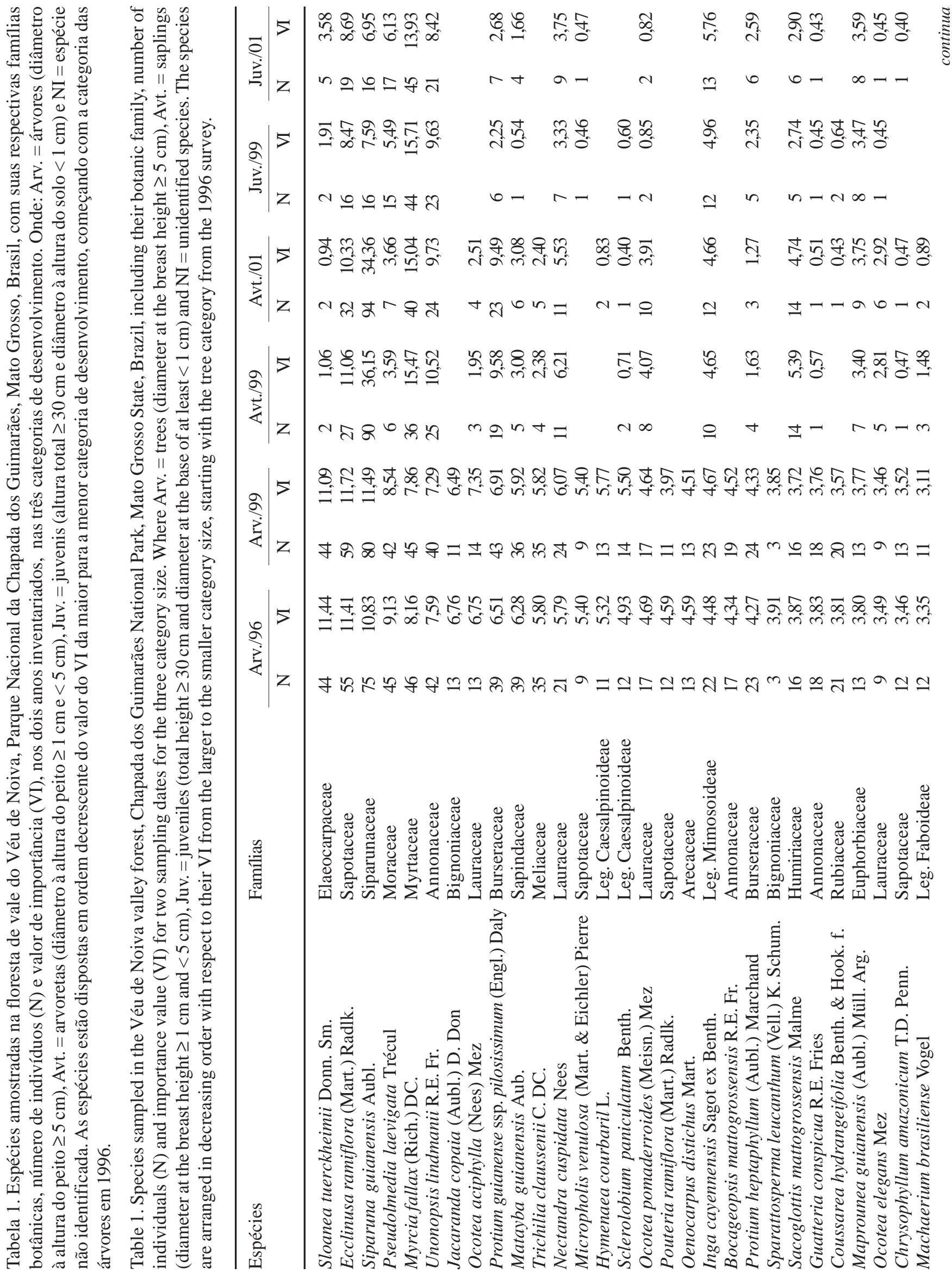




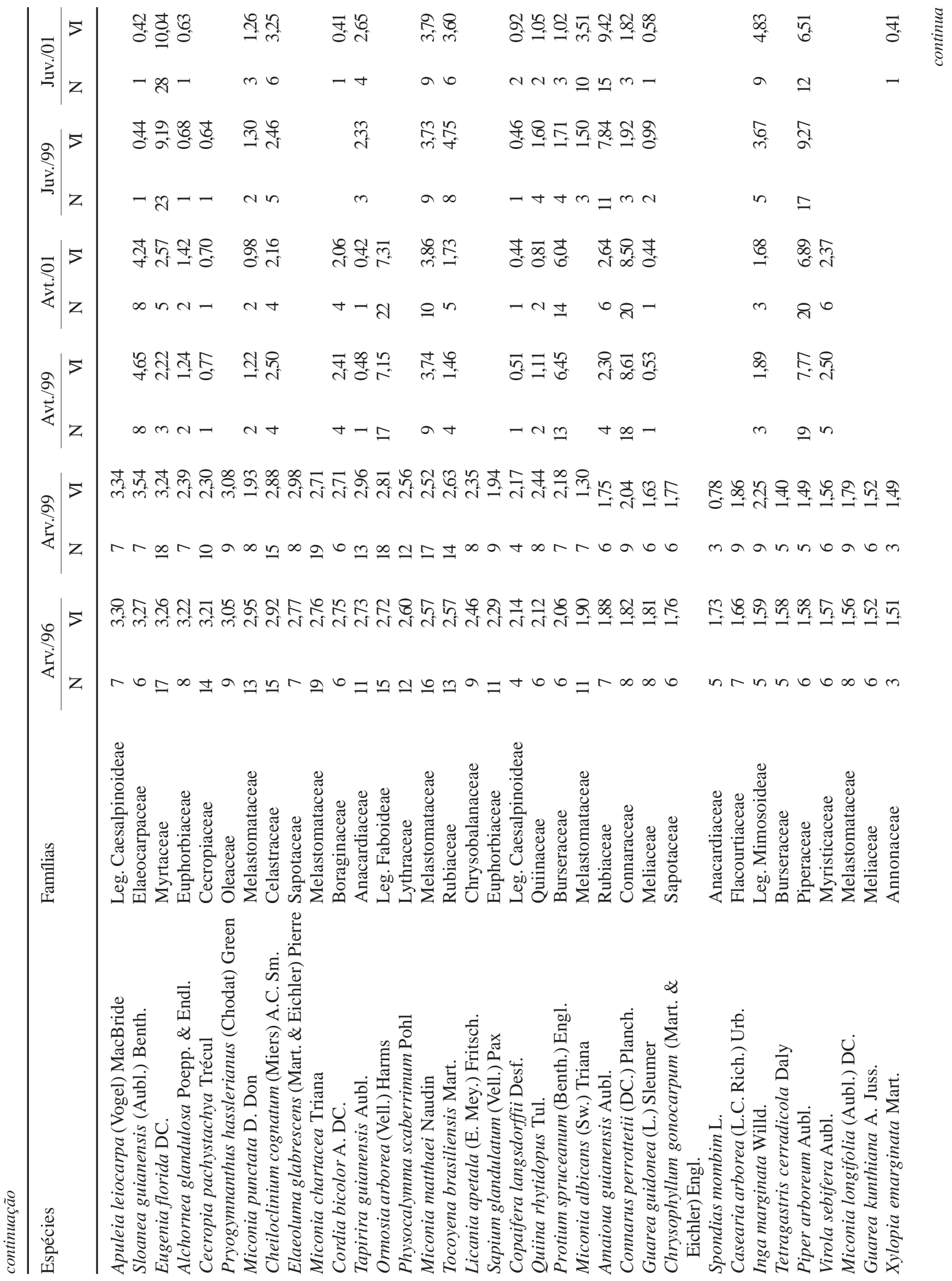




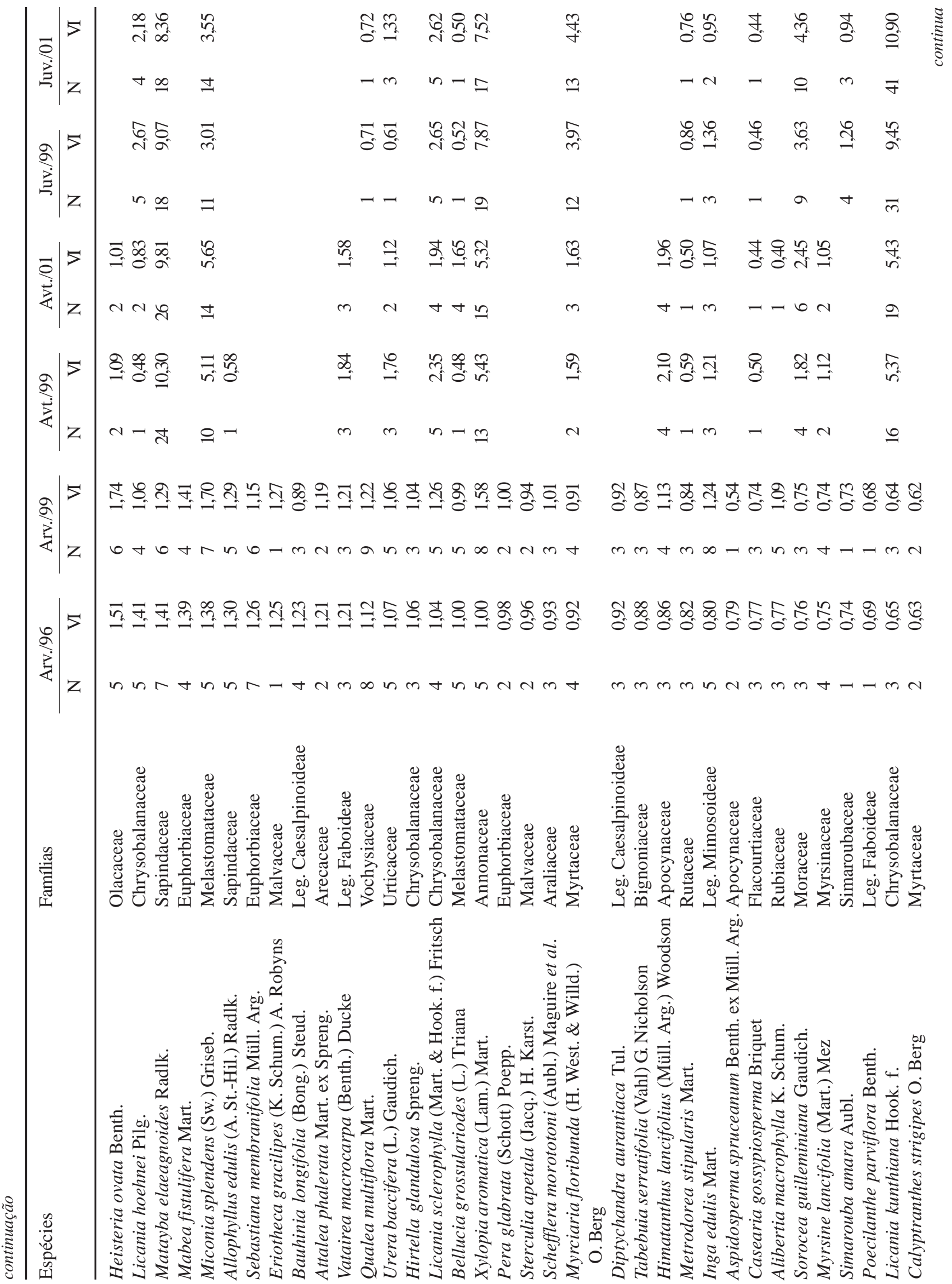




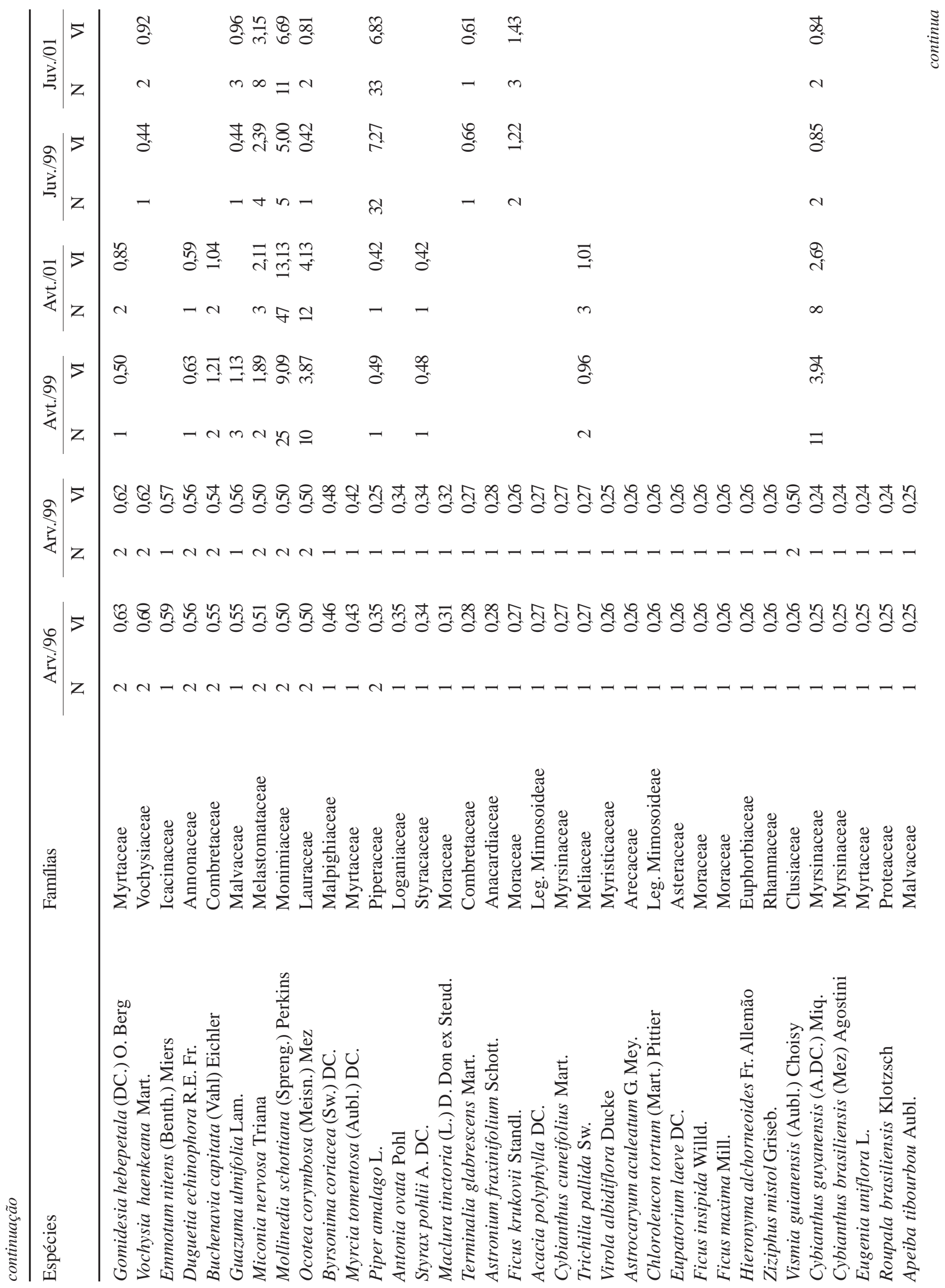









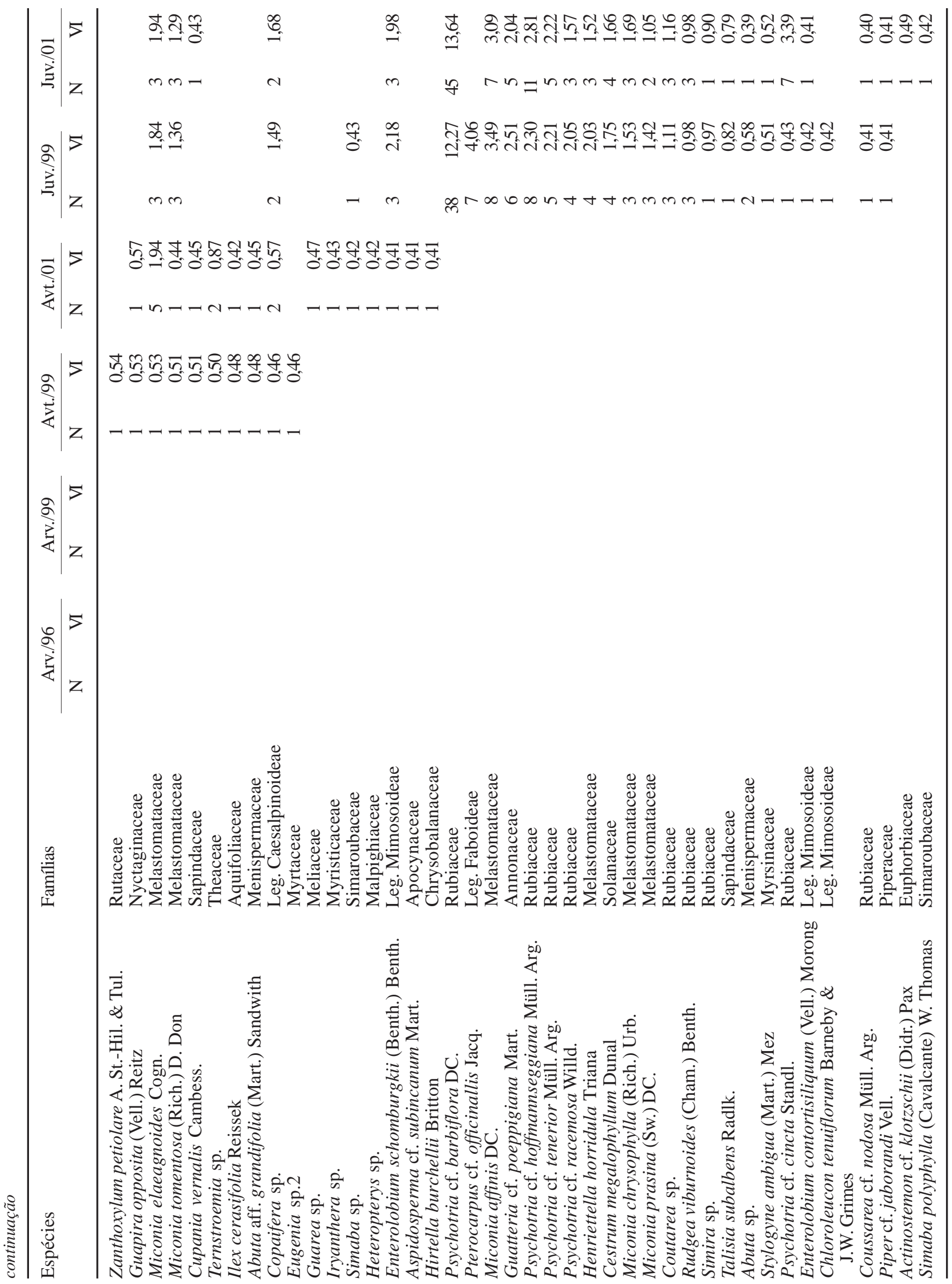


espécies exclusivas na categoria das árvores $(28,77 \%)$ e arvoretas + juvenis $(29,24 \%)$, excluindo o grupo da NI, foram menores que os $41,98 \%$ comuns às duas categorias. Esses resultados corroboram a idéia de que a diversidade de espécies em ecossistemas florestais é determinada não apenas pelas espécies que dominam o dossel ou o subosque, mas sim pela soma dos estratos que a compõem (Gilliam \& Roberts 1995).

O número de espécies amostradas com um único indivíduo, consideradas como espécies raras (sensu Martins 1991), foi alto durante o período estudado nas três categorias analisadas, perfazendo aproximadamente $27 \%$ entre as árvores, em 1996 e 1999; $32 \%$ e $34 \%$ nas arvoretas e $22 \%$ e $26 \%$ nas juvenis, em 1999 e 2001, respectivamente. Este comportamento confirma a tendência de que cerca de $1 / 4$ a um $1 / 3$ das espécies nas florestas tropicais são amostradas com baixa densidade (Hartshorn 1980) e próximo ao encontrado em outras florestas ripárias brasileiras, amostradas com semelhante intensidade amostral e critério de inclusão (Felfili 1994, Oliveira Filho et al. 1994a, b, Pagano et al. 1995, Felfili 1997a, Metzger et al. 1997, van den Berg \& Oliveira Filho 1999, Bertani et al. 2001, van den Berg
2001). Normalmente estas florestas são caracterizadas não só pela alta riqueza de espécies, mas também pela baixa densidade com que muitas delas são encontradas, o que possibilita a coexistência de inúmeras espécies por unidade de área (Hartshorn 1980). Assim, o elevado número de espécies amostradas com baixa densidade também contribuiu para a alta riqueza de espécies na floresta de vale do Véu de Noiva.

Os índices de diversidade e de eqüabilidade, calculados para as três categorias de desenvolvimento individualmente (tabela 2), foram altos se comparados com outros trabalhos realizados na região (Oliveira Filho \& Martins 1986, Oliveira Filho 1989, Monteiro 1994), ou mesmo com trabalhos realizados em outras florestas ripárias em diferentes regiões (Felfili 1994, Oliveira Filho et al. 1994a, b, van den Berg \& Oliveira Filho 1999). Essa elevada diversidade encontrada no Véu de Noiva está vinculada à sua localização geográfica em zona de tensão ecológica entre a Floresta Amazônica e o domínio do Cerrado e à grande heterogeneidade ambiental local (Pinto \& Oliveira Filho 1999). Incrementada, ainda, pelo caráter de conectividade que as florestas ripárias do Brasil Central apresentam, ligando as duas maiores

Tabela 2. Características florísticas e estruturais da comunidade arbórea da floresta de vale do Véu de Noiva, Parque Nacional da Chapada dos Guimarães, Mato Grosso, Brasil. Os valores são apresentados para os dois anos inventariados nas categorias das árvores (diâmetro à altura do peito $\geq 5 \mathrm{~cm}$ ), arvoretas (diâmetro à altura do peito $\geq 1 \mathrm{~cm} \mathrm{e}<5 \mathrm{~cm}$ ) e juvenis (altura total $\geq 30 \mathrm{~cm}$ e diâmetro à altura do solo $<1 \mathrm{~cm}$ ). H' = índice de diversidade de Shannon-Wiener, J' = índice de eqüabilidade de Pielou.

Table 2. Floristic and structural characteristics of the tree community in the Véu de Noiva valley forest, Chapada dos Guimarães National Park, Mato Grosso State, Brazil. The values are for both survey dates sampled in the category of trees (diameter at the breast height $\geq 5 \mathrm{~cm}$ ), saplings (diameter at the breast height $\geq 1 \mathrm{~cm}$ and $<5 \mathrm{~cm}$ ) and juveniles (total height $\geq 30 \mathrm{~cm}$ and diameter at the base of at least $<1 \mathrm{~cm})$. H' $=$ Shannon-Wiener diversity index, J'= Pielou's evenness index.

\begin{tabular}{|c|c|c|c|c|c|c|}
\hline \multirow{2}{*}{$\begin{array}{l}\text { Número de parcelas } \\
\text { Ano do inventário }\end{array}$} & \multicolumn{2}{|c|}{$\begin{array}{c}\text { Árvores } \\
18 \\
\end{array}$} & \multicolumn{2}{|c|}{$\begin{array}{c}\text { Arvoretas } \\
36\end{array}$} & \multicolumn{2}{|c|}{$\begin{array}{c}\text { Juvenis } \\
36\end{array}$} \\
\hline & 1996 & 1999 & 1999 & 2001 & 1999 & 2001 \\
\hline Densidade $^{*}$ & 1113 & 1138 & 3644 & 4265 & 3,28 & 3,50 \\
\hline Área basal $\left(\mathrm{m}^{2} \mathrm{ha}^{-1}\right)$ & 21,62 & 23,77 & 1,60 & 2,06 & - & - \\
\hline Mediana da altura (m) & 8,00 & 8,50 & 3,50 & 3,50 & 0,59 & 0,57 \\
\hline Mediana do DAP (cm) & 9,23 & 9,23 & 1,96 & 1,98 & - & - \\
\hline Número de espécies & 143 & 147 & 108 & 113 & 103 & 104 \\
\hline Número de famílias & 54 & 54 & 45 & 45 & 39 & 39 \\
\hline Perda de espécies (famílias) & $3(1)$ & 4(3) & $6(2)$ & & & \\
\hline Ganho de espécies (famílias) & $7(1)$ & $9(3)$ & $7(2)$ & & & \\
\hline H' (nats/ind.) & 4,33 & 4,34 & 3,94 & 3,95 & 3,98 & 4,02 \\
\hline $\mathrm{J}$ ' & 0,87 & 0,87 & 0,96 & 0,96 & 0,86 & 0,87 \\
\hline
\end{tabular}

*Árvores e arvoretas = indivíduos.ha ${ }^{-1}$ e Juvenis = indivíduos. $\mathrm{m}^{2-1}$. Para a categoria das juvenis não foram calculadas as áreas basais e as medianas do diâmetro representadas na tabela com traços (-). 
formações florestais brasileiras através dos corredores dendríticos (Oliveira Filho \& Ratter 1995) e apresentando componentes da flora Amazônia e Atlântica (Pinto \& Oliveira Filho 1999).

Mudanças na composição florística e diversidade de espécies - Para a categoria das árvores, em 1999, foram registradas sete novas espécies quando os dados são comparados com os do inventário de 1996 (Inga heterophylla, Isertia sp., Jacaranda cuspidifolia, Mouriri acutiflora, Rhamnidium elaeocarpum, Schefflera vinosa e Xylopia benthamii). Por outro lado, três espécies amostradas no primeiro inventário não foram registradas nas mesmas parcelas em 1999 (Aegiphila brachiata, Aegiphila lhotskiana e Banara tomentosa). Cabe ressaltar que das espécies com novos registros nas parcelas, quatro delas já haviam sido registradas na área em 1996, com DAP < 5 cm (Pinto \& Oliveira Filho 1999) e apenas Isertia sp., Jacaranda cuspidifolia e Xylopia benthamii foram registradas pela primeira vez nas parcelas. Entre as famílias, Verbenaceae que havia sido registrada no primeiro inventário, não o foi no segundo. Por outro lado, houve o registro de Memecylaceae nas parcelas na ocasião do segundo inventário; porém, esta já havia sido registrada em 1996, com DAP < $5 \mathrm{~cm}$.

Para as arvoretas, entre 1999 e 2001, foi registrado o ingresso de nove espécies novas (Aspidosperma $\mathrm{cf}$. subincanum, Enterolobium schomburgkii, Guarea sp., Hirtella burchellii, Iryanthera sp., Simaba sp., Heteropteris sp., Hymenaea courbaril e Coussarea hydrangeifolia). As três últimas já haviam sido amostradas em 1996 entre as árvores, com DAP < $5 \mathrm{~cm}$ (Pinto \& Oliveira Filho 1999) e Enterolobium schomburgkii havia sido amostrada, em 1999, como juvenil. Portanto, apenas cinco das nove espécies ainda não haviam sido registradas na área. Em contrapartida, o grupo das não identificadas - NI, assim como Allophyllus edulis, Guazuma ulmifolia e Zanthoxylum petiolare não foram amostradas como arvoretas em 2001; embora as duas primeiras continuassem presentes na área amostradas como árvores. Além do grupo das NI, não foram registradas, no segundo inventário, as famílias Rutaceae e Malvaceae; porém, ambas mantiveram-se representadas nas outras categorias de desenvolvimento. Por outro lado, foram registradas no interior das parcelas três novas famílias (Apocynaceae, Malpighiaceae e Simaroubaceae), apesar de já terem registro na área.

Para as juvenis, no período entre 1999 a 2001, foi registrada a ocorrência de sete novas espécies (Chrysophyllum amazonicum, Cordia bicolor,
Cupania vernalis, Roupala brasiliensis, Xylopia emarginata, Actinostemon cf. klotzschii e Simaba polyphylla) e duas novas famílias, Boraginaceae e Proteaceae. Apenas as duas últimas espécies ainda não haviam sido registradas na área. Por outro lado, não foram registradas seis espécies (Cecropia pachystachya, Coussarea hydrangeifolia, Sclerolobium paniculatum, Simaba sp., Pterocarpus cf. officinallis e Chloroleucon tenuiflorum) e duas famílias: Cecropiaceae e Leguminosae-Faboideae. Somente as duas últimas espécies deixaram, de fato, de ser registradas na área, as demais foram amostradas nas categorias de maior tamanho.

Comparando a listagem florística gerada a partir do inventário do componente arbóreo realizado dentro das parcelas em 1996 (Pinto \& Oliveira Filho 1999), acrescidas do levantamento da regeneração realizado em 1999 (Pinto 2002), com a listagem das espécies obtida a partir do inventário realizado em 1999 (para as árvores) e 2001 (para as arvoretas e juvenis), verificou-se que $3 \%$ das espécies registradas na primeira listagem não foram amostradas no segundo levantamento (três espécies amostradas na categoria das árvores, uma arvoreta e duas juvenis). Por outro lado, na ocasião do segundo levantamento, foram acrescidas oito espécies (5\% do total) na listagem florística (três espécies amostradas como árvores, cinco arvoretas e duas juvenis). Comparando estes resultados com outras florestas brasileiras isentas de interferências antrópicas de grandes proporções e amostradas com área semelhante ( 1,0 ha), o Véu de Noiva posiciona-se entre as que apresentaram menores perdas e maiores ganhos de espécies em inventários sucessivos (ver Felfili 1994, Pagano et al. 1995, Oliveira Filho et al. 1997, Pulz 1998, Cabral 1999, Guilherme 1999, Nascimento et al. 1999, Werneck et al. 2000, Bertani et al. 2001, van den Berg 2001). Exceto quando comparado com o cerrado sentido restrito, no Distrito Federal, onde a redução líquida na riqueza de espécies foi de $6,55 \%$ durante período de nove anos de estudo, em uma área com histórico de fogo (Felfili et al. 2000) e 13\% de aumento no número de espécies em uma área de 0,5 ha, num período de 2,4 anos e protegida do fogo por mais de 20 anos (Henriques \& Hay 2002). Portanto, em geral, o registro de novas espécies em inventários recorrentes numa mesma área, a partir de estudos com curtos intervalos entre as medições, está em torno de $3 \%$ a $13 \%$ e o desaparecimento entre $0 \%$ e $8 \%$.

Provavelmente, a baixa perda e o alto ganho de espécies entre os dois inventários realizados no Véu de Noiva, em relação aos estudos citados acima, deve-se 
ao fato de que os demais levantamentos concentraram se apenas na categoria das árvores (DAP $\geq 5 \mathrm{~cm}$ ). Certamente, isso fez com que a diferença entre o número de espécies amostradas no primeiro e no segundo inventários fosse alta ("pseudo-extinção"), visto que muitas das espécies que haviam sido amostrada no estrato arbóreo na ocasião do primeiro inventário e não foram registradas no segundo continuam presentes na comunidade, porém com tamanho inferior ao critério de inclusão, ou mesmo, presentes apenas no banco de plântulas (Kellman et al. 1998, Felfili et al. 2000, Werneck et al. 2000). De acordo com Swaine et al. (1987), as mudanças na composição de espécies em florestas tropicais livres de interferência antrópica e de grandes distúrbios geralmente são muito baixas. Além disso, o registro de ocorrência de novas espécies na área, ou o não registro de outras, por ocasião de inventários recorrentes, está relacionado às espécies localmente pouco abundantes (Kellman et al. 1998, Nascimento et al. 1999, Felfili et al. 2000, Werneck et al. 2000, van den Berg 2001), na maioria dos casos $N<3$. Essa situação, de fato, foi confirmada na floresta de vale do Véu de Noiva, pois apenas uma espécie entre as árvores e duas espécies entre as juvenis foram amostradas pela primeira vez, no segundo inventário, com mais de três indivíduos. Entre as árvores foram registrados dois novos indivíduos de Jacaranda cuspidifolia em 1999, enquanto, entre as juvenis, Pterocarpus cf. officinallis apresentou perda de sete indivíduos e Roupala brasiliensis o registro de cinco novos indivíduos.

Embora o cálculo do índice de diversidade seja afetado pelo número de espécies e pela distribuição de abundância entre as espécies (Magurran 1988), as mudanças encontradas na riqueza e na densidade parecem ter sido pequenas a ponto de não alterar este índice durante o período estudado. Com isso, as diferenças nos índices entre os anos de amostragem não foram significativas em nenhuma das categorias de desenvolvimento (teste $t, P>0,05$ ).

Felfili et al. (2000) comentam que o equilíbrio dinâmico normalmente registrado em estudos de dinâmica de comunidades vegetacionais se deve às flutuações cíclicas, alternando períodos com alta mortalidade seguidos por outros com alto recrutamento, o que mantém a estrutura e a composição florística da comunidade como aparentemente estáveis. Felfili et al. (2000) propõem, para o Cerrado, que os monitoramentos de longo prazo sejam feitos inicialmente com mensurações regulares em curtos intervalos de tempo, três anos, com a finalidade de captar tais flutuações pois, em intervalos mais amplos, as oscilações se diluem no tempo e deixam de ser perceptíveis. No Véu de Noiva o período de três anos para o estrato arbóreo e dois anos para as arvoretas e juvenis parece ter sido curto para tornar significativas as diferenças florísticas e, conseqüentemente, os índices de diversidade. Somente com sucessivas avaliações de longo prazo, com intervalos regulares, poderemos fazer afirmações com propriedade sobre as mudanças florísticas dessa comunidade.

Mudança na estrutura fitossociológica - Em florestas tropicais com mais de 100 espécies é comum cerca de $5 \%$ a $10 \%$ das espécies representarem $50 \%$ ou mais do VI total (Hartshorn 1980), enquanto que nos ambientes ripários do Brasil Central, aproximadamente 1/5 das espécies detêm mais da metade do número de indivíduos (Felfili et al. 2001). Esse último comportamento se repetiu no Véu de Noiva, pois $20 \%$ das espécies amostradas entre as árvores somaram aproximadamente $56 \%$ do VI total e $58 \%$ do número de indivíduos. Entre as arvoretas esta mesma porcentagem de espécies representa cerca de $62 \%$ do VI e $67 \%$ dos indivíduos e entre as juvenis corresponde, em média, a $57 \%$ do VI e $62 \%$ do total de indivíduos. Todavia, a participação individual de cada população pode ser alterada com o tempo (Felfili 1995a).

A posição hierárquica das espécies com base no valor de importância entre os anos inventariados para cada categoria de desenvolvimento mudou pouco (tabela 1). Analisando conjuntamente o grupo das espécies mais importante com base no VI, em cada categoria, ou seja, 20\% do número de espécies, verificouse que entre as árvores $(N=29) 11$ espécies apresentaram mudanças positivas na sua posição hierárquica, 13 sofreram redução e cinco mantiveram a posição original. Entre as arvoretas $(N=22)$, foram observadas seis mudanças positivas, nove negativas e sete mantiveram a posição original, ao passo que entre as juvenis $(N=20)$ foram registradas 11 espécies com mudanças positivas, quatro negativas e cinco não mudaram.

Henriques \& Hay (2002) afirmam que as espécies mais abundantes recrutam mais indivíduos do que as espécies raras. Por outro lado, as espécies de maior VI são as mais hábeis para explorar os recursos disponíveis nas áreas (Felfili 1994) e, portanto, é de se esperar que estas espécies, na ausência de grandes distúrbios, alterem pouco sua participação na estruturação da comunidade. Felfili (1995b) comenta que mesmo as espécies mais abundantes sendo sujeitas às maiores taxas de mortalidade, elas também apresentam maior taxa de recrutamento, mantendo assim a sua supremacia 
na área. Entretanto, pequenas alterações na densidade das espécies mais abundantes, como encontrado no Véu de Noiva, também foram observadas em outras áreas (ver Felfili 1994, Pagano et al. 1995, Oliveira Filho et al. 1997, Cabral 1999, Guilherme 1999, Felfili et al. 2000, Werneck et al. 2000, Bertani et al. 2001, van den Berg 2001). As mudanças na densidade e na área basal das espécies mais abundantes, mesmo que pequenas, indicam que a estrutura fitossociológica da comunidade possui um caráter dinâmico e as espécies dominantes podem ser alteradas com o tempo, conforme sugere Felfili (1994), mudando assim a estrutura fitossociológica da comunidade como um todo.

Mudanças na distribuição de freqüência nas classes de diâmetro e de altura - A categoria das árvores seguiu o padrão típico das florestas tropicais, com distribuição de frequiência do tipo sigmóide para as classes de altura e exponencial negativa ('J-invertido'), para as classes de diâmetros (figura 2A). Este tipo de distribuição de freqüência permite inferir que na comunidade há indivíduos, independente da espécie, ocupando de forma diferenciada o espaço vertical em função da forma de vida e/ou do grupo ecológico que pertençam tais espécies. As arvoretas e juvenis apresentaram distribuições tendendo a linear negativa, principalmente em diâmetro para as arvoretas (figura 2B) e altura para as juvenis (figura 2C). De acordo com Schiavini et al. (2001), as perturbações sofridas pela floresta ficam impressas na estrutura das populações e são refletidas na distribuição diamétrica e de altura da comunidade. Estas interferências são perceptíveis ao longo de décadas (Oliveira Filho et al. 1997), ou mesmo centenas de anos (Cabral 1999). Na floresta de vale do Véu de Noiva como não há registro histórico de interferência antrópica direta, as distribuições de freqüências em altura e em diâmetro são resultados da própria dinâmica natural da comunidade.

As distribuições de freqüências nas classes de altura e de diâmetro (figura 2) não diferiram estatisticamente entre os dois anos inventariados em nenhuma das três categorias de desenvolvimento amostradas (Kolmogorov-Smirnov, $p>0,05$ ). Além disso, as mudanças nos valores das medianas em altura e em diâmetro foram mínimas nas três categorias (tabela 2). $\mathrm{O}$ fato das distribuições de freqüências e os valores das medianas em diâmetro e em altura não terem sido significativamente diferentes demonstra que a estrutura vertical e a estrutura diamétrica da floresta mantiveram o padrão registrado no primeiro levantamento. Esse comportamento também foi observado em duas florestas de galeria no Distrito Federal (Felfili 1997b, c).
Entretanto, essa virtual estabilidade florística e estrutural encontrada não deve levar à interpretação de que a floresta do Véu de Noiva é uma comunidade estática. Certamente a composição florística e a estrutura da floresta estão em constante alteração, provavelmente em função das mudanças nas condições ambientais, bem como em resposta aos distúrbios naturais de baixa intensidade que ocorrem na área, tais como a queda de árvores isoladas, como sugeriram Pinto \& Oliveira Filho (1999).

Mudanças na densidade e área basal - As mudanças estruturais entre os anos inventariados foram positivas nas três categorias de desenvolvimento. Os menores aumentos foram registrados entre as árvores e os maiores entre as arvoretas, tanto em densidade como em área basal (tabela 2), sendo significativo apenas em termos de densidade na categoria das arvoretas $\left(\chi^{2}=48,76 ; P<0,0001\right)$.

$\mathrm{O}$ aumento na densidade e na área basal na comunidade como um todo é um indicativo de que a floresta está em fase de acúmulo de biomassa, talvez como resultado das flutuações cíclicas, alternando entre períodos de expansão volumétrica seguidos por períodos de retração, conforme mencionado por Hubbell \& Foster (1990), Felfili (1995a), Kellman et al. (1998) e Pulz (1998). Neste caso, estas flutuações estariam sendo intensificadas em função da floresta de vale do Véu de Noiva ser influenciada por distúrbios naturais de pequena intensidade, por exemplo, abertura de clareiras de pequeno porte. Estes distúrbios estariam sendo favorecidos pela pouca profundidade do solo, pela topografia íngreme e pela ação de ventos canalizados que freqüentemente incidem no vale, resultando em instabilidade das árvores de grande porte e, conseqüentemente, danos às plantas do dossel intermediário e do subosque (Pinto \& Oliveira Filho 1999).

Aparentemente os benefícios oriundos da abertura do dossel superaram os malefícios, pois as mudanças na densidade e área basal foram todas positivas, provavelmente em resposta às condições favoráveis de luminosidade e redução na competição intra e interespecífica. A mesma situação foi descrita por van den Berg (2001), em relação aos indivíduos do dossel intermediário e inferior em uma floresta ripária no sul de Minas Gerais.

As mudanças em densidade e área basal foram variadas dentro e entre as categorias de desenvolvimento, indicando que as mudanças são aleatórias e multidirecionais (Pinto 2002). Isso demonstra que o estágio de desenvolvimento em que a vegetação 


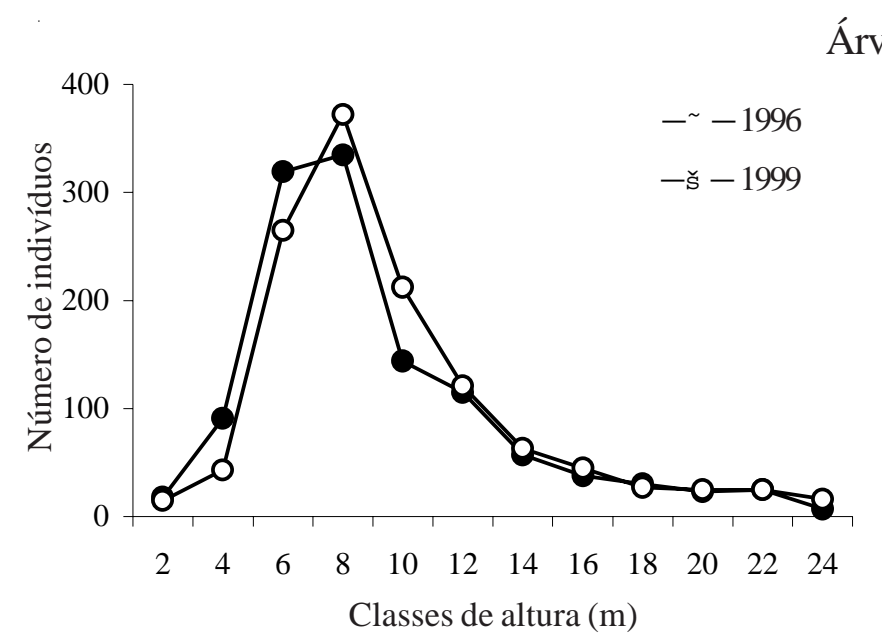

Árvores

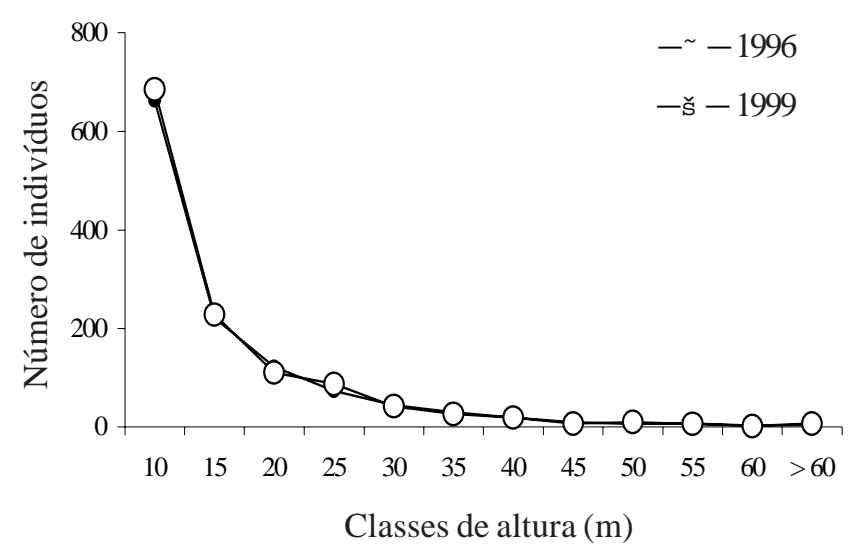

Árvoretas


Juvenis

$\mathrm{C}$

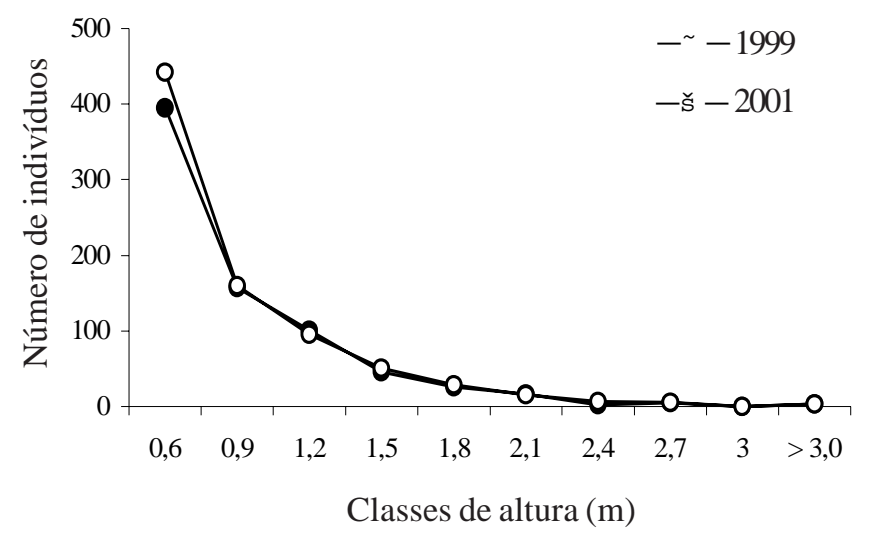

Figura 2. Histograma de distribuição de frequiência nas classes de altura (direita) e de diâmetro (esquerda), dos indivíduos amostrados na floresta de vale do Véu de Noiva, Parque Nacional da Chapada dos Guimarães, Mato Grosso, Brasil. (A) Distribuição das árvores - diâmetro à altura do peito $\geq 5 \mathrm{~cm}$; (B) distribuição das arvoretas - diâmetro à altura do peito $\geq 1 \mathrm{~cm}$ $\mathrm{e}<5 \mathrm{~cm}$ e $(\mathrm{C})$ distribuição das juvenis - altura total $\geq 30 \mathrm{~cm}$ e diâmetro à altura do solo $<1 \mathrm{~cm}$.

Figure 2. Frequency distribution by height classes (right) and diameter classes (left) of sampled individuals in the Véu de Noiva valley forest, Chapada dos Guimarães National Park, Mato Grosso State, Brazil. Trees with diameter at the breast height $\geq 5 \mathrm{~cm}$ (A); saplings, with diameter at the breast height $\geq 1 \mathrm{~cm}$ and $<5 \mathrm{~cm}$ (B); and juveniles with total height $\geq 30 \mathrm{~cm}$ and diameter at the base of at least $<1 \mathrm{~cm}(\mathrm{C})$. 
é avaliada pode influenciar na intensidade destas mudanças. Neste caso, a categoria intermediária (arvoretas), foi a que apresentou as maiores mudanças, tanto florística como estrutural, contrariando a idéia de que tais características mudam pouco nas florestas tropicais.

Agradecimentos - Aos professores Ary T. Oliveira Filho (DCF/UFLA), Ivan Schiavini (IB/UFU), Jeanine M. Felfili (EFL/UnB) e José Felipe Ribeiro (Embrapa/CPAC) pelas sugestões. Ao amigo Eddie Lenza de Oliveira e a Fabiana de Góis Aquino pela leitura crítica do manuscrito. Aos amigos do curso de Engenharia Florestal (UFMT), pela ajuda nos trabalhos de campo. Ao Prof. Ary T. Oliveira Filho (DCF/UFLA) e ao técnico Libério Amorim Neto (IB/UFMT) pelo auxílio na identificação botânica. Ao CNPq pela concessão da bolsa de estudo ao primeiro autor.

\section{Referências bibliográficas}

APG II. 2003. An update of the Angiosperm Phylogeny Group classification for the orders and families of flowering plants: APG II. Botanical Journal of the Linnean Society 141:399-439.

BERTANI, D.F., RODRIGUES, R.R., BATISTA, J.L.F. \& SHEPHERD, G.J. 2001. Análise temporal da heterogeneidade florística e estrutural em uma floresta ribeirinha. Revista Brasileira de Botânica 24:11-23.

BRASIL. 1982. Projeto RadamBrasil: folhas SE 21 - Corumbáe SD 21 - Chapada dos Guimarães; geomorfologia, pedologia, vegetação e uso potencial da terra. Departamento Nacional de Produção Mineral, Rio de Janeiro.

CABRAL, V.A.R. 1999. Dinâmica de um fragmento de mata ciliar do Rio Grande em Bom Sucesso - Minas Gerais. Dissertação de mestrado, Universidade Federal de Lavras, Lavras.

EITEN, G. 1994. Vegetação do cerrado. In Cerrado: caracterização, ocupação e perspectivas (M.N. Pinto, ed.). Editora UnB, Brasília, p.17-73.

FELFILI, J.M. 1994. Floristic composition and phytosociology of the gallery forest alongside the Gama stream in Brasília, DF, Brazil. Revista Brasileira de Botânica 17:1-11.

FELFILI, J.M. 1995a. Growth, recruitment and mortality in the Gama gallery forets in Central Brazil over a six-year period (1985-1991). Journal of Tropical Ecology 11:67-83.

FELFILI, J.M. 1995b. Diversity, structure and dynamic of a gallery forest in Central Brazil. Vegetatio 117:1-15.

FELFILI, J.M. 1997a. Dynamics of the natural regeneration in the Gama gallery forest in Central Brazil. Forest Ecology and Management 91:235-245.
FELFILI, J.M. 1997b. Comparison of dynamics of two galery forests in Central Brazil. In Proceedings of International Symposium on Assessment and Monitoring of Forests in Tropical Dry Regions With Special Reference to Gallery Forests (J. Imaña-Encinas \& C. Kleinn, coords.). Universidade de Brasília, Brasília, p.115-124.

FELFILI, J.M. 1997c. Diameter and height distribution in a gallery forest tree community and some of its main species in Central Brazil over a six year period (1988-1991). Revista Brasileira de Botânica 20:155-162.

FELFILI, J.M. 2000. Crescimento, recrutamento e mortalidade nas matas de galeria do Planalto Central. In Tópicos Atuais em Botânica (T.B. Cavalcanti \& B.M.T. Walter, coords.). Embrapa - Cenargen, Brasília, p.152-158.

FELFILI, J.M., REZENDE,A.V., SILVAJUNIOR, M.C. \& SILVA, M.A. 2000. Changes in the floristic composition of cerrado sensu stricto in Brazil over a nine-year period. Journal of Tropical Ecology 16:579-590.

FELFILI, J.M., FRANCO, A.C., FAGG, C.W. \& SOUSA-SILVA, J.C. 2001. Desenvolvimento inicial de espécies de mata de galeria. In Cerrado: caracterização e recuperação de matas de galeria (J.F. Ribeiro, C.E.L. Fonseca \& J.C. Sousa-Silva, eds.). EMBRAPA - CPAC, Planaltina, p.779-811.

GILLIAM, F.S. \& ROBERTS, M.R. 1995. Impacts of forest manegement on plant diversity. Ecological Applications 5:911-912.

GUILHERME, F.A.G. 1999. Efeitos do regime de inundação e de bambus na dinâmica da comunidade arbórea de um fragmento de floresta semidecídua no Sul de Minas Gerais. Dissertação de mestrado, Universidade Federal de Lavras, Lavras.

HARTSHORN, G.S. 1980. Neotropical forests dynamics. Biotropica 12:23-30.

HARTSHORN, G.S. 1990. An overview of neotropical forest dynamics. In Four Neotropical Rainforests (A.H. Gentry, ed.). Yale University Press, New Haven, p.585-599.

HENRIQUES, R.P.B. \& HAY, J.D.V. 2002. Patterns and dynamics of plant populations. In The Cerrados of Brazil: ecology and natural history of a Neotropical Savanna (P.S. Oliveira \& R.J. Marquiz, eds.). Columbia University Press, New York, p.140-158.

HUBBELL, S.P. \& FOSTER, R.B. 1990. Structure, dynamics and equilibrium status of old-growth forest on Barro Colorado Island. In Four Neotropical Rainforests (A.H. Gentry, ed.). Yale University Press, New Haven, p.522-541.

IBAMA. 1994. Plano de ação emergencial: documento e informações básicas do Parque Nacional da Chapada dos Guimarães. Instituto Brasileiro do Meio Ambiente e dos Recursos Naturais Renováveis - Ibama/MT, Cuiabá.

KELLMAN, M., TACKABERRY, R. \& RIGG, L. 1998. Structure and function in two tropical gallery forest communities: implications for forest conservation in fragmented sytems. Journal of Applied Ecology 35:195-206. 
LIEBERMAN, M., LIEBERMAN, D. \& PERALTA, R. 1989. Forest are not just swiss cheese: canopy stereogeometry of non-gaps in tropical florests. Ecology 70:550-552.

MAGURRAN, E.A. 1988. Ecological diversity and its measurement. Princeton University Press, Princeton.

MARTINS, F.R. 1991. Estrutura de uma floresta mesófila. Editora da Unicamp, Campinas.

METZGER, J.P., BERNACCI, L.C. \& GOLDENBERG, R. 1997. Patterns of tree species diversity in riparian forest fragments of different widths (SE Brazil). Plant Ecology 133:135-152.

MONTEIRO, J.R.B. 1994. Composição florística e estrutura de uma floresta mesófila semidecídua no município de Chapada dos Guimarães - MT. Dissertação de mestrado, Universidade Estadual de Campinas, Campinas.

MUELLER-DOMBOIS, D. \& ELLENBERG, H. 1974. Aims and methods of vegetation ecology. John Wiley and Sons, New York.

NAIMAN, R.J. \& DÉCAMPS, H. 1997. The ecology of interfaces: riparian zones. Annual Review Ecology Systematic 28:621-658.

NASCIMENTO, H.E.M., DIAS, A.S., TABANEZ, A.A.J. \& VIANA, V.M. 1999. Estrutura e dinâmica de populações arbóreas de um fragmento de floresta estacional semidecidual na região de Piracicaba, SP. Revista Brasileira de Biologia 59:329-342.

OLIVEIRA FILHO, A.T. 1989. Composição florística e estrutura comunitária da floresta de galeria do Córrego da Paciência, Cuiabá (MT). Acta Botanica Brasilica 3:91-112.

OLIVEIRA FILHO, A.T. 2002. Dynamics of tree communities and populations in riparian and upland forest in Brazil. In $45^{\text {th }}$ Symposium of the International Association for Vegetation Science (V.D.P. Pillar, coord.). Departamento de ecologia e botânica, UFRGS e International Association for Vegetation Science, Porto Alegre, p.12.

OLIVEIRA FILHO, A.T. \& MARTINS, F.R. 1986. Distribuição, caracterização e composição florística das formações vegetais da região da Salgadeira, na Chapada dos Guimarães (MT). Revista Brasileira de Botânica 9:207-223.

OLIVEIRAFILHO, A.T. \& RATTER, J.A. 1995. A study of the origin of Central Brazilian forests by the analysis of plant species distribution patterns. Edinburgh Journal of Botany 52:141-194.

OLIVEIRAFILHO, A.T., VILELA, A.E., GAVILANES, M.L. \& CARVALHO, D.A. 1994a. Effect of soil and topography on the distribution of tree species in a tropical riverine forest in south-eastern Brazil. Journal of Tropical Ecology 10:483-508.

OLIVEIRAFILHO, A.T., VILELA, E.A., CARVALHO, D.A. \& GAVILANES, M.L. 1994b. Differentiation of streamside and upland vegetation in an area of montana semideciduous forest in southeastern Brazil. Flora 189:287-305.
OLIVEIRAFILHO, A.T., MELLO, J.M. \& SCOLFORO, J.R.S. 1997. Effects of past disturbance and edges on tree community structure and dynamic within a fragment of tropical semideciduous forest in south-eastern Brazil over a five-year period (1987-1992). Plant Ecology 131:45-66.

PAGANO, S.R., LEITÃOFILHO, H.F. \& CAVASSAN, O. 1995. Variação temporal da composição florística e estrutura fitossociológica de uma floresta mesófila semidecíua Rio Claro - estado de São Paulo. Revista Brasileira de Biologia 55:241-258.

PHILLIPS, O.L. \& GENTRY, A.H. 1994. Increasing turnover through time in tropical forests. Science 263:954-958.

PHILLIPS, O.L., HALL, P., GENTRY, A.H., SAWIER, S.A. \& VASQUEZ, R. 1994. Dynamics and species richness of tropical rain forest. Proceeding of the National Academy of Science (USA) 91:2805-2809.

PINTO, J.R.R. 1997. Levantamento florístico, estrutura da comunidade arbóreo-arbustiva e suas correlações com as variáveis ambientais em uma floresta de vale no Parque Nacional da Chapada dos Guimarães, Mato Grosso. Dissertação de mestrado, Universidade Federal de Lavras, Lavras.

PINTO, J.R.R. 2002. Dinâmica da comunidade arbóreoarbustiva em uma floresta de vale no Parque Nacional da Chapada dos Guimarães, Mato Grosso. Tese de doutorado, Universidade de Brasília, Brasília.

PINTO, J.R.R. \& OLIVEIRAFILHO, A.T. 1999. Perfil florístico da comunidade arbórea de uma floresta de vale no Parque Nacional da Chapada dos Guimarães, Mato Grosso, Brasil. Revista Brasileira de Botânica 22:53-67.

PULZ, F.A. 1998. Estudo de dinâmica e a modelagem da estrutura diamétrica de uma floresta semidecídua montana na região de Lavras - MG. Dissertação de mestrado, Universidade Federal de Lavras, Lavras.

REES, M., CONDIT, R., CRAWLEY, M., PACALA, S. \& TILMAN, D. 2001. Long-term studies of vegetation dynamics. Science 293:650-655.

RIBEIRO, J.F. \& WALTER, B.M.T. 2001. As matas de galeria no contexto do bioma Cerrado. In Cerrado: caracterização e recuperação de Matas de Galeria (J.F. Ribeiro, C.E.L. Fonseca \& J.C. Souza-Silva, eds.). Embrapa - CPAC, Planaltina, p.29-47.

RICHARDS, P.W. 1996. The tropical rainforest. $2^{\text {th }}$ ed. Cambridge University Press, Cambridge.

RODRIGUES, R.R. \& NAVE, A.G. 2000. Heterogeneidade florística das matas ciliares. In Matas Ciliares: conservação e recuperação (R.R. Rodrigues \& H.F. Leitão Filho, eds.). EDUSP, São Paulo, p.45-71.

SCHIAVINI, I., RESENDE, J.C.F. \& AQUINO, F.G. 2001. Dinâmica de populações de espécies arbóreas em matas de galeria e mata mesófila na margem do Ribeirão Panga, MG. In Cerrado: caracterização e recuperação de Matas de Galeria (J.F. Ribeiro, C.E.L. Fonseca \& J.C. SouzaSilva, eds.). Embrapa - CPAC, Planaltina, p.267-299. 
SHUGART, H.H. 1984. A theory of forest dynamics: the ecolocal implications of forest succession models. Springer-Verlag, New York.

SWAINE, M.D. 1990. Population dynamics of tree species in tropical forests. In Tropical forest: botanical dynamics, speciation and diversity (L.B. Holm-Nielsen, I.C. Nielsen $\&$ H. Balslev, eds.). Academic Press, London, p.101-110.

SWAINE, M.D., LIEBERMAN, D. \& PUTZ, F.E. 1987. The dynamics of tree populations in tropical forest: a review. Journal of Tropical Ecology 3:359-366.

TILMAN, D., NAEEM, S., KNOPS, J., REICH, P., SIEMANN, E., WEDIN, D., RITCHIE, M. \& LAWTON, J. 1997. Biodiversity and ecosystem properties. Science 278:1866-1867.

VAN DEN BERG, E. 2001. Variáveis ambientais e a dinâmica estrutural e populacional de uma floresta de galeria em Itutinga, MG. Tese de doutorado, Universidade Estadual de Campinas, Campinas.
VAN DEN BERG, E. \& OLIVEIRA FILHO, A.T. 1999. Spatial partitioning among tree species within an area of tropical montane gallery forest in south-eastern Brazil. Flora 194:249-266.

VELOSO, H.P., RANGEL FILHO, A.L.R. \& LIMA, J.C.A. 1991. Classificação da vegetação brasileira adaptada a um sistema universal. Fundação IBGE, Rio de Janeiro.

WERNECK, M.S., FRANCESCHINELLI, E.V. \& TAMEIRÃO NETO, E. 2000. Mudança na composição florística e estrutura de uma floresta decídua durante um período de quatro anos (1994-1998), na região do triângulo mineiro, MG. Revista Brasileira de Botânica 23:399-411.

WHITMORE, T.C. 1990. An introduction to tropical rainforest. Oxford University Press, Oxford.

ZAR, J. H. 1998. Biostatistical analysis, $4^{\text {th }}$ ed. Prentice-Hall, New Jersey. 